Methods Kenya has Prevention of MTCT Technical Working Group with members from Ministry of Health, PEPFAR agencies, UN family, implementing partners focusing on guideline and policy development. From single dose nevirapine in 2005 to Option A in 2010, Kenya is rolling out new guidelines with Option $\mathrm{B}+$, elimination framework, mentor mother programmes, health systems strengthening activities, community strategies, private partnerships, and maternal/child health integration of anti-retroviral (ARV) therapy. Appointment diaries, mobile telephones, home visits are retention strategies. Longitudinal antenatal registers, mother-baby booklet, HIV-Exposed Infant (HEI) registers for easy identification and tracking of mothers and infants used. HEI are identified and tested at 6-8 weeks through PCR. Capacity building, infrastructure, supportive supervision, commodity security, safe infant feeding are monitored. EID dashboard (website) shows EID results from all PCR labs in real-time.

Results Kenya demonstrates tremendous progress from 2005 to 2012: PMTCT sites from 926 to 4,500; pregnant women counselled and tested for HIV from 318,000 to 1.2 million (80\% coverage); ARV prophylaxis from $52 \%$ to $90 \%$ of HIV-positives identified; CD4 access from $<50 \%$ to $>72 \%$; over $57 \%$ attending $4+$ antenatal visits. Exclusive breastfeeding for 6 months increased from 3.2\% to $32 \%$ (KDHS). The number of infants tested by PCR increased from 4,500 in 2006 to 64,000 in 2011. PCR positivity has dropped from $11.2 \%$ (2010) to 7.6\% (2011) and 5.2\% (2012) at 6-week testing of infants. Conclusion Use of more efficacious regimen including Option B+, integration of services, implementation of new guidelines and eMTCT framework should enable Kenya to attain a transmission rate less than $5 \%$ by 2015 .

\section{P6.015 EXPLORING EXPERIENCES WITH COMMUNITY BASED PROMOTION OF EXCLUSIVE BREASTFEEDING IN THE CONTEXT OF HIV IN THE RURAL MALAWI}

doi:10.1136/sextrans-2013-051184.1169

'A K Bula, 'C McCourt, ${ }^{2} \mathrm{M}$ Magadi, ${ }^{3}$ S Lewycka, ${ }^{3} \mathrm{~T}$ Phiri. 'School of Health Sciences, City University London, London, UK; ${ }^{2}$ City University, London, UK; ${ }^{3}$ MaiMwana Project, Mchinji, Malawi

Background Exclusive breastfeeding (EBF) for 6 months is ranked as the most effective way of feeding babies in Sub-Saharan Africa where HIV positive women are poor and infant mortality is high. However, mixed feeding is common, increasing risks of infant death due to malnutrition, diarrhoeal, HIV, and other infectious diseases. Community-based Interventions has proved effective in promoting EBF in poor settings. Results of MaiMwana intervention in Malawi are underway. However, there was little attention on the needs of HIV positive women during the design and conduct of these interventions. The aim of this study was to explore the importance and experiences with implementing the intervention in Mchinji, Malawi in the context of HIV.

Method We purposively selected and conducted qualitative indepth interviews with 39 key informants in Mchinji, Malawi between January to August 2012 using a pre-designed interview guide. Responses were analysed by Framework analysis. The study was approved by the Malawi National Health Sciences and City University London research ethics committees.

Results HIV positive and negative women were supportive of the intervention for continuity of infant feeding counselling as volunteers spend more time with them. HIV positive women appreciated the visit because they had to tell their storey to someone. However, some did not disclose their status due to fear of stigma and abandonment by family members present during the visit, making it difficult for counsellors to effectively provide counselling on EBF. Service users also find the intervention as not being cost-effective due to lack of time and money to travel to the hospital for other health services.
Conclusion It is important for projects to consider the needs of HIV positive women when designing community-based interventions to reduce HIV transmission and deaths among infants. Integration of services is desired considering the levels of poverty and distance to health facilities.

\section{P6.016 FACTORS ASSOCIATED WITH FREQUENT ALCOHOL USE AMONG FEMALE SEX WORKERS IN THREE HIGH PREVALENCE STATES OF INDIA: FINDINGS FROM A BIO- BEHAVIOURAL SURVEY}

doi:10.1136/sextrans-2013-051184.1170

'D Yadav, 'S Ramanathan, 'P Goswami, 'L Ramakrishnan, 'S Sen, 'B George, ${ }^{2} \mathrm{R}$ Paranjape, ${ }^{3} \mathrm{~T}$ Subramanian, ${ }^{4} \mathrm{H}$ Rachakulla. ${ }^{1} \mathrm{FHI}$ 360, New Delhi, India; ${ }^{2}$ National AIDS Research Institute (NARI), Pune, India; ${ }^{3}$ National Institute of Epidemiology (NIE), Chennai, India; ${ }^{4}$ National Institute of Nutrition (NIN), Hyderabad, India

Background HIV prevalence among female sex workers (FSW) is around eight times higher than general population in India. There is limited information on alcohol use and related risk factors among FSWs in the country. To inform HIV prevention interventions, we assessed the patterns of alcohol use among FSWs and its association with background characteristics and other risk behaviour using data from a bio-behavioural survey (2009-2010).

Methods 7,806 women aged 18 years or older who sold sex in exchange of cash at least once in past month were recruited from Andhra Pradesh, Tamil Nadu and Maharashtra states in India using two-stage time location cluster sampling. Behavioural information was collected through structured questionnaires, blood and urine specimens were tested for HIV and other STIs.

Results About one fourth (26\%) of FSWs reported consuming alcohol daily or at least once a week, and termed as 'frequent' alcohol users. Among them, four-fifth were aged above 24 years, $68 \%$ illiterate, $63 \%$ currently married, $29 \%$ had experienced physical violence, $58 \%$ were in sex work profession for more than five years and $78 \%$ were using condom consistently with regular clients. The frequent alcohol users were more likely to be in sex work for five plus years (AOR: 1.42, $\mathrm{p}<0.05$ ), had 10 or more clients per week (AOR: $1.53, p<0.05$ ), experienced physical violence (AOR: 1.64, $p<0.05$ ), were in-debt (AOR: $1.71, p<0.05)$ and reported anal sex with clients (AOR: 1.81, $\mathrm{p}<0.05)$. However, frequent alcohol use was not associated with increased STIs (NG/CT/Syphilis) and HIV prevalence.

Conclusion These findings suggest the frequent alcohol use is associated with other high risk behaviour that can increase vulnerabilities for HIV and STI. Therefore targeted interventions needs to address alcohol use and associated factors, which could positively impact HIV prevention interventions among FSWs in India.

\section{P6.017 THE CHALLENGES OF INTRODUCING A SOFTWARE-BASED INTERVENTION TO INCREASE STI AND HIV TESTING AMONG GAY AND BISEXUAL MEN}

doi:10.1136/sextrans-2013-051184.1171

'D Callander, ${ }^{2} \mathrm{C}$ Bourne, ${ }^{3} \mathrm{~V}$ Ramanathan, ${ }^{4} \mathrm{~J}$ de Wit, ${ }^{1} \mathrm{R}$ Guy. ${ }^{1}$ The Kirby Institute of Infection and Immunity in Society, Sydney, Australia; ${ }^{2}$ Sydney Sexual Health Centre, Sydney, Australia; ${ }^{3}$ Central Sydney GP Network, Sydney, Australia; ${ }^{4}$ National Centre in HIV Social Research, Sydney, Australia

Background Information technology is being used increasingly in sexual health services to improve clinical efficiencies and sexual health management. While past research has demonstrated the effectiveness of such interventions, little attention has been paid to their use in general practise. We describe the challenges of introducing a sexual health information technology package in nine general practise clinics with medium to high case loads of gay or bisexual men. 